Proceedings of the 2002 IEEE

International Conference on Robotics \& Automation

Washington, DC - May 2002

\title{
Modeling, Scheduling, and Prediction for Wafer Fabrication: Queueing Colored Petri-Net and GA Based Approach
}

\author{
An-Chih Huang, Li-Chen Fu, Ming-Hung Lin, and Shun-Yu Lin \\ Department of Computer Science and Information Engineering \\ National Taiwan University, Taipei, Taiwan, R.O.C.
}

\begin{abstract}
In this paper, we propose a modeling tool named QCPN (Queueing Colored Petri Net). The main idea of this tool is to combine the original CTPN (Colored Timed Petri Net) with the queueing systems. With QCPN model, we can predict the delivery date of any specific product under some scheduling policies efficiently and rather precisely. In the scheduling phase. we use the GA based approach to search for the optimal combination of a number of heuristic rules. This $Q C P N$ based $G A$ scheduler can greatly reduce the computation time so as to meet the need for a rapidly changing environment.
\end{abstract}

\section{Introduction}

In recent years, semiconductor manufacturing industry grows rapidity and it has become one of the most competitive fields. Due to equipment uncertainty, product diversity, process intricacy, and ever improving technologies, the semiconductor manufacturing is perhaps the most complicated manufacturing process found today.

This paper addresses the problem of prediction and scheduling. If we want to meet requirements of customers, we have to precisely estimate the cycle time of all orders as well as other performance measures (work in process, machine utilization) first. Based on these measures, the manager or fab engineers can control the operation and the manufacturing times easily, and the customers can know the date of lot delivery beforehand. Thus, prediction becomes an extremely important and challenging task. There are many papers [1][2] which proposed to model manufacturing facilities to predict product cycle time. Among them, the mathematical model based on Queueing Theory is the most commonly used one, which is also adopted in this paper. In order to accomplish the task of prediction, a wafer fab model based on QCPN (Queueing Colored Petri Net)[10] is proposed in this paper. This model not only helps us to evaluate these performance measures, but also helps us to develop and evaluate our scheduler easily. Petri Net has played an important role in modeling field. It has been developed into a powerful tool for discrete event systems; particularly in manufacturing systems. Many extensions of Petri net were introduced, such as colored Petri net [3], and timed Petri net which were widely used in manufacturing systems. The colored timed Petri net (CTPN) is used to model the furnace in the IC wafer fabrication [3] and in the whole wafer fabrication manufacturing system [4][11]. Jeng et al. [5] reported a project of applying Petri net methodologies to detailed modeling, qualitative analysis, and performance evaluation of the etching area in an IC wafer fabrication system bcated in the Science Based Industrial Park in Hsin Chu, Taiwan.

There are many papers on planning and scheduling. Wein [6] has investigated the effect on both input $\mathrm{re}$ lease policies and dispatching policies, though via a detailed simulation study. Most conventional heuristic procedures use a priority rule. Traditional dispatching methods like FIFO, critical ratio, least slack time and least queue time have been proposed [7]. In recent years, an interest in using probabilistic search methods to solve job shop problem has been growing rapidly. Lee et al. [8] combined the machine learning and genetic algorithm in the job shop scheduling.

The organization of this paper is described as follows. In Section 2, the definitions of the proposed Queueing colored Petri net (QCPN) are revealed here, and the detailed systematic method of QCPN model is discussed in Section 3. In Section 4, we first describe the problem of prediction. Then, we describe the way of delivery prediction when some priority-based dispatching rules are adopted. Some popular scheduling problems and lot released control policies used in wafer fabrication are discussed first in Section 5 Then, a GA embedded search method over the QCPN model is employed. In Section 6, we briefly describe the implementation of the simulation tool. In addition, we demonstrate the examples of using the proposed mechanism and analyze the performance. Finally, conclusions and future research are provided in Section 7 and 8.

\section{Queueing Colored Petri-net}

In our method of combining Petri Net and Queueing network, we introduced a special kind of transition, namely, "queueing transition" into the original Colored Timed Petri Net to represent the role of queueing system. The following is the definition of QCPN.

\subsection{Definition of Queueing Colored Petri-net}

A Queueing Colored Petri Net (QTPN) is a bipartite graph, defined by a 12-tuple, CTPN $=\left(P_{i}, P_{c}, T_{i}, T_{n}\right.$, $T_{t}, T_{s}, T_{n}, T_{q}, C, I, O, M$ ), where

\footnotetext{
$P_{i}$ set of immediate places:

$P_{c} \quad$ set of communication places:

$T_{i} \quad$ set of immediate transitions;

$T_{n}$ set of mapping transitions;

$T_{1}$ set of timed transitions;

$T_{s}$ set of stochastic transitions;
} 
$T_{n}$ set of macro transitions;

$T_{q}$ set of queueing transitions;

$C$ color set of transition and place;

I input functions;

$O$ output functions;

$M$ the marking.

An elaboration of these elements is the same as the ordinary CTPN[3][6]. The icon definition of CTPN is illustrated in Fig. 1

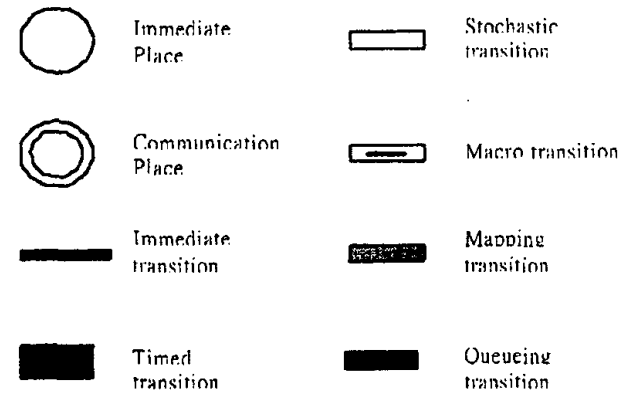

Figure 1 Icon definition of CTPN

As mentioned, we introduced six kinds of transitions and two kinds of places into our model. The brief description of these transitions and places are given as follows.

Immediate places: Immediate places are the same as the ordinary places but without the time attribute.

$\square$ Communication places: The interconnection between different modules is achieved by the communication place [3].

$\square$ Macro transitions: Each macro transition contains a module of the model, and this module combines arcs, places and transitions [3].

$\square$ Immediate transitions: Immediate transitions are the same as the ordinary transitions in CPN (Colored Petri-net).

$\square$ Mapping transitions: After firing the mapping transitions, the token corresponding to a certain color that enables this type of transition is transferred to another place with the predefined color.

$\square$ Timed transitions: Timed transitions describe the time properties of resources or time delay.

$\square$ Stochastic transitions: The transition times may belong to one of several distributions in stochastic transitions.

$\square$ Queueing transitions: A token that enables the queueing transition $q$ with respect to the color $q c$ will be assigned a queueing time (waiting time). Each queueing transition can be embedded with a priority queueing model. The wating time for the queueing transition can be calculated by the queueing model. After the queueing time delay, this transition can be fired.

In Figure 2(a), the original service queue is illustrated by CTPN. With our proposed QCPN, this Esource contention subset can be illustrated in Figure 2(b). In this figure, original place resource in Figure 2(a) is removed. It is replaced by a queueing transition enter.
The queueing transitions are embedded with the priority queueing model. Thus, this queueing model can calculate the delay (waiting) time of the queueing transition.

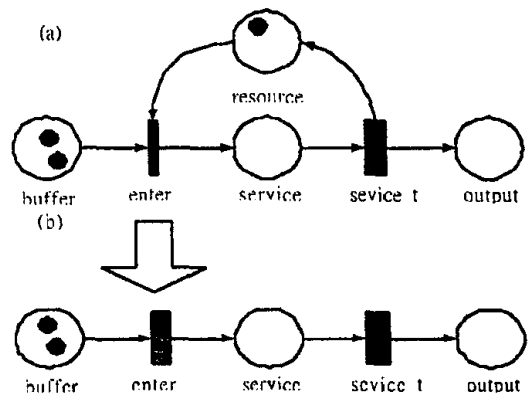

Figure 2 (a) Original resource contention (b) QCPN resource contention

\subsection{Waiting Time of Queueing Transition}

The tool group in a semiconductor foundry is a typical priority queue. Because of operation reasons, lots are classified into several priority classes. The urgent lots are given the highest priority for operation. We can use $\mathrm{G} / \mathrm{G} / 1 /$ Priority queueing system to calculate waiting time. The mean waiting time is approximated by incorporating an adjustment factor $j$ into $\mathrm{M} / \mathrm{G} / 1 /$ priority model [1]. Denote $W_{p}$ as waiting time of token of (priority) class $p, \lambda_{1}$ as arrival rate of token of class $p, \overline{x_{i}^{2}}$ as $2^{\text {nd }}$-order moment of wafer processing time of token of class $p$. Then,

$$
W_{p}=\frac{\sum_{i=1}^{p} \frac{\lambda_{i} \overline{x_{i}^{2}} j_{i}}{2}}{\left(1-\sigma_{p}\right)\left(1-\sigma_{p+1}\right)} \text { where } j_{i} \equiv \frac{c_{u, k}^{2}+c_{s . k}^{2}}{c_{s, k}^{2}+1},
$$

$c_{a, k}^{2}$ and $c_{s, k}^{2}$ are the squares of variation. coefficients of the inter arrivals and processing times of the lots with priority $p$. The parameter $c_{a, k}^{2}$ and $c_{s, k}^{2}$ can be obtained from the historical running data of the wafer fab.

\section{Wafer Processing Model}

We use he proposed QCPN to model the whole wafer manufacturing system.

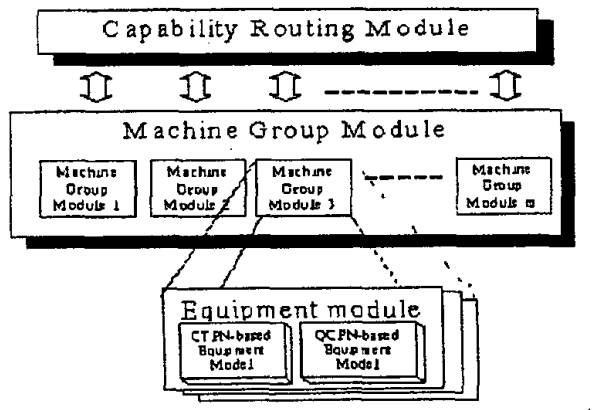

Figure 3 Conceptual architecture of the wafer fab model 
The details of the model including Capability Routing Module, Machine Group Module and Equipment Module are described in the following sections. Figure 3 illustrates the conceptual architecture of the proposed model.

\section{Capability Routing Module}

The main purpose of Capability Routing Module is to model the logical process flow of the semiconductor manufacturing systems.

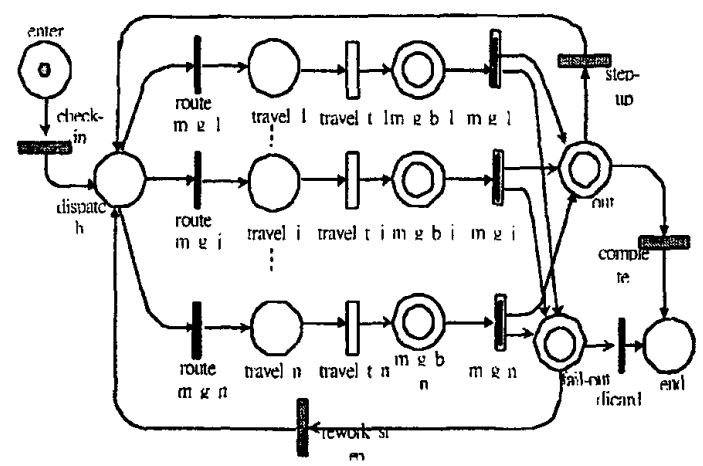

Figure 4 Capability routing module

\section{Machine Group Module}

The machine group module contains one or more identical machines (or processing units). This module is responsible for dispatching the lot to an appropriate equipment module.

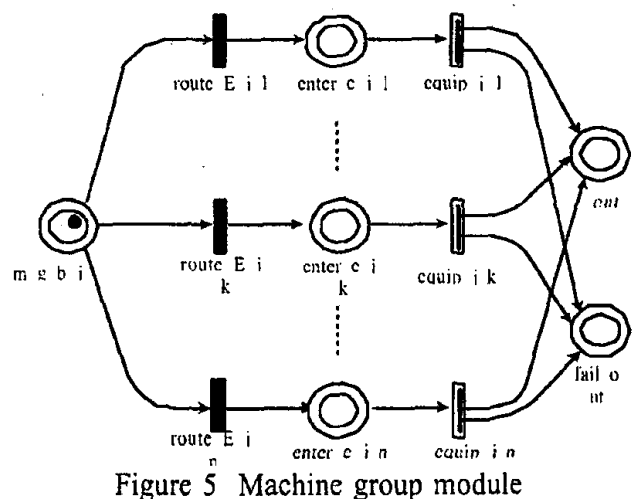

\section{Equipment module}

The QCPN equipment model is illustrated in Figure 6 . The waiting time of the tokens (lots) is calculated using the priority queueing model of the queueing transition.

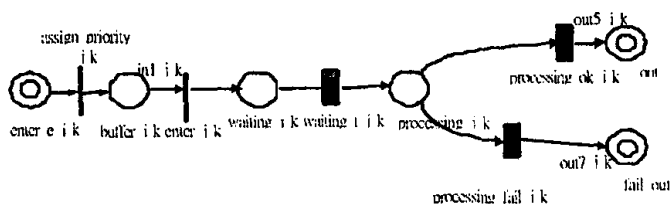

Figure 6 QCPN based equipment module

The machine failure mechanism and periodical maintenance subnet is not modeled in Petri-net in Figure
6. Such occasional events can be modeled as the lots that directly arrive at the highest priority queue [1] in priority queueing model mentioned above.

\section{Delivery Prediction}

In order to provide more information to the customer, and to help them to make ordering decisions, we need to estimate some performance measures precisely and efficiently. In this section, we use the wafer processing model described in Section 3 to predict the delivery date of the order.

\subsection{Necessary Input Parameters}

Since QCPN is a semi-simulation tool, we can construct our system model and simulate it. Thus, some input parameters are needed to simulate the system. We describe these parameters in the following items.

Current system state: This information includes the current status of the lots and the machines.

$\square$ Future Demands: These are the potential orders, which will be released in the future.

Current rules: These are scheduling rules that will be adopted in the future.

$\square$ Other parameters: These parameters include the probability of machine failure, rework probability of lot, and some other minor parameters that will affect the precision

The predictor can also answer some what-if questions by simulating result.

\subsection{Priority Assigning Method for General Priority-Based Rules}

As mentioned, we use the priority queueing model as a part of our modeling tool. When various scheduling rules are used, we have to decide which priority class the lot should be assigned to.

After the highest and lowest value of the lots in fab are found, we can assign the lot to different priority class according to the following equation.

$$
\begin{gathered}
C=\left[\frac{p-V_{\min }}{V_{\max }-V_{\min }} \times P\right]\left(V_{\max }>p>V_{\min }\right) \\
C=P\left(p>V_{\max }\right) \\
C=1\left(p<V_{\min }\right)
\end{gathered}
$$

where $P$ is the number of priority classes, $V_{\max }$ is the highest priority value among lots, $V_{\min }$ is the lowest priority value among lots, $p$ is the priority value of the target lot, and $C$ is the priority class that this lot should be assigned to. Note that the value of $V_{\max }$ and $V_{\min }$ need to be updated periodically.

The proposed QCPN model based predictor which can predict some performance measures such as lot due date over the corresponding QCPN model. Since the prediction must be performed under some kind of scheduling rules, the priority assigning method proposed here can combine the scheduling rules with the prediction. 


\section{Wafer Fab Scheduler}

Most semiconductor manufacturing companies suffer from high WIP level and long cycle times. Hence, it is important to develop a production scheduling system that can help minimize cycle time and WIP as well as to meet the due date constraints.

\subsection{Genetic Algorithm}

There are two sub-problems in the scheduling problem. First, what searching method do we apply to optimize the scheduling results? Next, how do we evaluate the performance of each scheduling policy? For the first sub-problem, we apply a Genetic Algorithm. On the other hand, for the second sub-problem, we use mathematical analysis based on our QCPN model to address the performance evaluation. In other words, we evaluate the objective measures of our fitness function through the analyzing procedure of the proposed QCPN model. The general structure of a genetic algorithm is given in L. M. Wein [9].

\subsection{Mixed Dispatch Rule}

The main idea of this scheduler in this paper is to use a GA algorithm to find the appropriate lot release rule, machine selecting rule and dispatching rule. In addition, we try to mix some dispatching rules together and use GA search to find the appropriate mixed mode of each machine. This method, first normalize the:value of priority-base dispatching rules used in our method into the same range. Then, it uses different combinations of these rules to generate different execution sequence of lots on each machine.

Different priority-based rules may have different ranges of values. The main goal of this transformation (or normalization) is to transform these priority values into the same range. Note that in some rules the smaller priority value represents the higher priority of a lot, so we need to make the value negative. The transformation equation will be

$$
C=\frac{p-V_{\min }}{V_{\max }-V_{\min }}
$$

where $V_{\max }\left(V_{\min }\right)$ is the highest (lowest) priority value among lots, $C$ is the transformed value, and $p$ is the target lot priority (the value to be transformed). Then, the priority value of different rule can be transformed into values between $0 \sim 1$. Note that each priority-based dispatching rule has its own $V_{\text {max }}$ and $V_{\min }$, so that we can track the range of values of each rule.

We use EDD, CR, SRPT, LS, and Penalty rules in this paper, and each rule has a discrete parameter range, say, from 0 to 3 . We multiply this parameter with the transformed priority value of each rule, and then add these values up. The summation is divided by the sum of the parameters, and then the priority of the mixed rule is obtained. Whether some comb inations of these rules may be useful or not, the GA search algorithm will eliminate those "bad" combinations, and reserve only good combinations of chromosomes after generations.

\subsection{Chromosome Representation}

There are three types of genes in our chromosome: $g_{l}, g_{m}$, and $g_{d}$, which denotes lot release policy, machine selection rule, and dispatch rule respectively. $g_{d}=(a, b, c, d, e)$ is a five-tuple where

$\square \quad$ a: selected $p$ arameter for SRPT

$\square$ b: selected parameter for EDD

$\square$ c: selected parameter for CR

$\square$ d: selected parameter for SLACK

$\square$ e: selected parameter for Penalty

For each rule in $g_{l}$ and $g_{m}$, it is described simply as follows:
UNIF: Uniform Distribution
CONWIP: Constant WIP
$\square$ WR: Workload Regulating
$\square$ UTIL_L: Lowest Utilization
$\square$ SQL: Shortest Queue Length
SEPT: Shortest expected processing time Table 1.

The rules we selected for gene codes are listed in Table 1 Gene codes

\begin{tabular}{|l|l|l|l|l|l||}
\hline $\begin{array}{l}\text { Lot Release Pol i- } \\
\text { cies }\end{array}$ & $\begin{array}{l}\text { Machine Selecting } \\
\text { Rule }\end{array}$ & \multicolumn{2}{l|}{$\begin{array}{l}\text { Dispatch } \\
\text { Rules }\end{array}$} \\
\hline Name & Code & Name & Code & Name & Code \\
\hline UNIF & 1 & UTIL_L & 1 & SRPT & $0 \sim 3$ \\
\hline CONWIP & 2 & SQL & 2 & EDD & $0 \sim 3$ \\
\hline WR & 3 & SEPT & 3 & CR & $0 \sim 3$ \\
\hline & & & & SLACK & $0 \sim 3$ \\
\hline & & & & Penalty & $0 \sim 3$ \\
\hline
\end{tabular}

These gene codes are directly related to our QCPN model. The dispatch rule is related to QCPN model by selecting an appropriate priority queue for each lot. Since the transformed priority value is between 0 and 1 , we can calculate the appropriate priority queue by $C=[p, \times P]$, where $p_{i}$ is the transformed priority value of the lot, and $C$ is the priority class (queue) this lot should be assigned to. The waiting time can be calculated by $M / G / 1 /$ priority queueing model described in Section 2.

The chromosomes can be created after the genes are defined. The main goal of the GA search algorithm is to find a good chromosome that can satisfy the predefined requirements such as shorter cycle time and higher meet-due-date rate.

\subsection{Fitness Function}

In common situations, we have multiple objective measures. How to integrate these functions becomes an important issue. One of the methods is to combine them into a scalar fitness solution by weighted-sum approach: Here, we explain the function definition as follows: ( $c$ is a chromosome (i.e., solution))

$$
f(c)=w_{1} \cdot f_{1}(c)+w_{2} \cdot f_{2}(c)+w_{3} \cdot f_{3}(c)
$$

where $f_{j}$ is the score for mean cycle time, 
$f_{2}$ is the score for throughput rate,

$f_{3}$ is the score for the loss due to the number of orders which failed to meet due date.

The value of these scores is obtained from the QCPN wafer fab model. We code any possible schedule solutions into the chromosomes. The scheduler simulates the quality of the individual chromosome in detail through the PN platform to guide the proper genetic evolution to produce a good enough schedule.

\section{Experiment}

To verify the effectiveness of the proposed work, we build a virtual fab model based on the data from a semiconductor fab in Hsinchu, Taiwan. However, for business protection, all the data are scrambled, but the scale is approximately kept. In this model, there are totally 477 machines in 43 machine groups. The machines in each machine group may range from 3 to 45 . There are two production lines in this model. The first production line is a reentrant line comprising 416 operations, and the second production line comprising 322 operations. Since the number of machines in the fab and that of operations in the production line are large, the complexity in simulation, prediction and scheduling is enormously increased.

Some machine groups in this model contain batch-processing machines. The parameters such as MTTF (Mean Time to Failure), MTTR (Mean Time to Repair) and scheduled maintenance are also included in this model. Finally, scheduled maintenance means that the machine will be maintained after $\mathrm{n}$ times of operations.

\subsection{Experiment Results}

In this section, we design some experiments to evaluate the performance of our predictor and scheduler. In these experiments, work in process of the fab is not empty in the beginning of evaluation. We generate the information of these lots by taking a snapshot of the simulation model already in steady state. The period of evaluation is 3000 hours (about 45 months) if we did not mention. Similarly, the average rate of lot release is 6.4 lots per day. Our experiments are performed on the mixed production line, and the proportion of two types of lots is $1: 1$.

\section{The Precision of The Predictor}

In this experiment, we arbitrarily choose three orders and compare their delivery prediction values with simulation ones. Each order containes two lots with different type (flow), and the interval of order release is 8 hours. In order to verify the performance of the predictor on different dispatching ules, the four rules SRPT, EDD, CR and LS are chosen for comparison. Table 2 shows the configuration of these three orders.

Table 2 The configuration of three chosen order

\begin{tabular}{|c|c|c|}
\hline Name & Release Time (Hour) & Due Date (Hour) \\
\hline OrderP22 & 168 & 988 \\
\hline
\end{tabular}

\begin{tabular}{||l|l|l|}
\hline OrderP42 & 328 & 1148.71 \\
\hline OrderP62 & 488 & 1308.71 \\
\hline
\end{tabular}

Figure 7 illustrates the results of this experiment.

The accuracy of prediction ranges from $90 \%$ to $98 \%$, depending on the rule used.

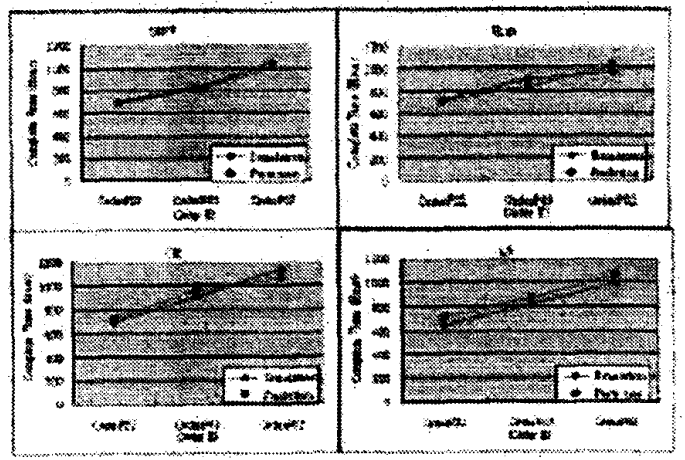

Figure 7 Complete time comparison

\section{The Performance of The GA Sche duler}

Here, we compare the performance measures of our scheduler with other priority-based heuristic rules first. The period of evaluation is 3000 hours, and the interval of order release is 7.5 hours. The due date of the orders is the order release time plus 640 hours, and the penalty is randomly generated values ranges from 0 to 10 . The GA parameters we use to search for a better chromosome are listed as follows:

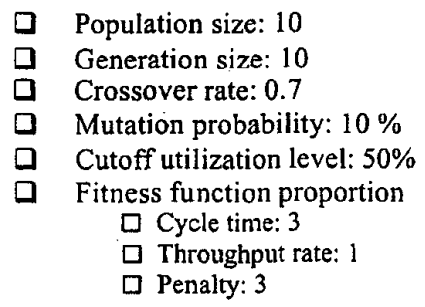

The scheduler is based on the QCPN model to evaluate the performance measures. The computation time of GA scheduler is about $2 \sim 3$ minutes.

Form Figure 7, we found that the proposed QCPN based predictor have high precision when different priority based rules are used. For the predictor, it can also achieve some requirements such as performing quick-responding prediction whereas maintaining good preciseness.

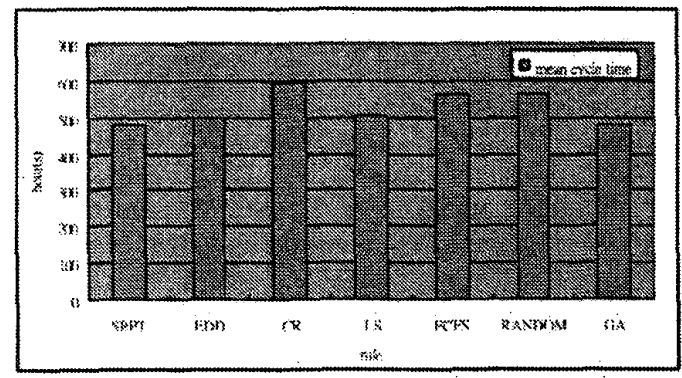

Figure 8 Cycle time comparison of the scheduler 


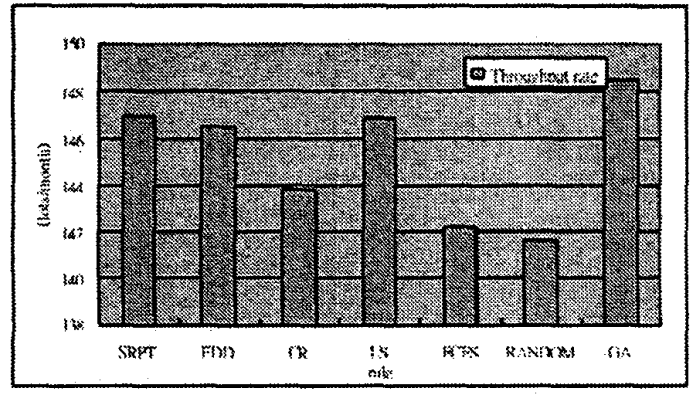

Figure 9 Throughput rate comparison of the scheduler

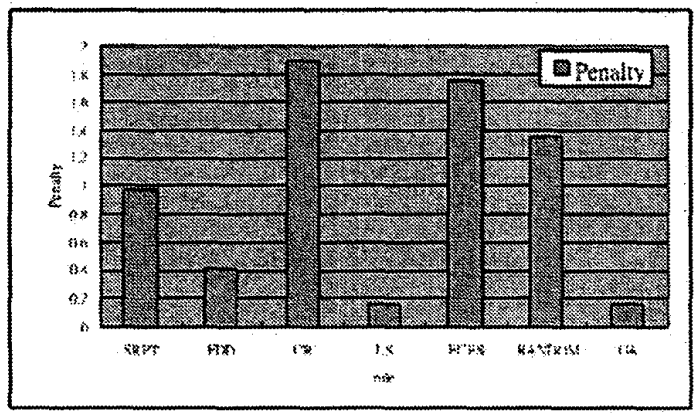

Figure 10 Penalty comparison of the scheduler

From Figure 8 to 10 , we found that the proposed GA scheduler performs much better than other priority based rule. It has a lower mean cycle time for lots spent in the fab, a higher throughput rate for a fab, and a lower value of penalty. As a result, the proposed GA scheduler has a significant impact on wafer fab scheduling by providing obvious performance improvements even though the fab has a mixed production.

\section{Conclusion}

In this paper, we proposed one novel modeling tool, named, QCPN (Queueing Colored Petri Net). The main idea of this tool is to combine the original CTPN with the queueing systems. With QCPN model, we can predict the product delivery date precisely and efficiently under some scheduling policies. We achieve this goal by periodically changing the priority of each lot. Since most of the scheduling rules (dispatching rules) are priority based ones, changing the priority of each lot can reflect the behavior of these rules. In the scheduling phase, we use the GA based approach to search for the optimal combination of a number of heuristic rules. This approach can reduce the solution space to find the good solution more quickly. So this GA scheduler can serve the rapidly changing enviro nment well.

\section{Discussion and Future Research}

Applying QCPN as the underlying model in our simulator saves much computation time. For GA, which needs many runs of simulation to obtain its solution, this advantage is much more valuable. There will be some loss of the accuracy of the simulation from using the queueing transition, however, we can reduce its impact by some mechanism such as updating the arrival rate periodically. Since there is no single rule which always performs very well, the use of mixed rule shows its merit. In the future research, we will keep on the topic of the mechanisms making the arrival rate more accurate and better methods to mix the rules.

\section{Reference}

[1] D. P. Connors, G. E. Feigin, and D. D. Yao, " A Queueing Network Model for Semiconductor Manufacturing," IEEE Transactions on Semiconductor Manufacturing, vol. 9, no. 3, pp. 412-427, 1996.

[2] P. Buchholz, "A New Approach Combining Simulation and Randomization for the Analysis of Large Continuous Time Markov Chains.", ACM Transactions on Modeling and Computer Simulation, Vol. 8, No. 2, April 1998, pp. 194-222.

[3] M. H. Lin and L. C. Fu, "Modeling, Analysis, Simulation, and Control of Semiconductor Manufacturing Systems: A Generalized Stochastic Colored-Timed Petri-Net Approach," IEEE International Conference on Systems Man, and Cybernetics, 1999.

[4] M. C. Zhou and M. D. Jeng, "Modeling, Analysis, Simulation, Scheduling and Control of Semiconductor Manufacturing Systems: A Petri Net Approach," IEEE Transactions on Semiconductor Manufacturing, vol. 11, no. 3, pp. 333-357, 1998.

[5] M. D. Jeng, X. Xie, and S. W. Chou, "Modeling, Qualitative Analysis, and Performance Evaluation of the Etching Area in an IC Wafer Fabrication System Using Petri Nets," IEEE Transactions on Semiconductor Manufacturing, vol. 11, no. 3, pp. 358-373, 1998.

[6] L. M. Wein, "Scheduling Semiconductor Wafer Fabrication," IEEE Transactions on Semiconductor Manufacturing, vol. 1, no. 3, pp. 1]5-130, 1988.

[7] K. R. Baker, "Sequencing Rules and Due-Date Assignments in Job Shop." Management Science, Vol. 30, No. 9, pp.1093-1104, Sep. 1984

[8] C. Y. Lee, S. Piramuthu, and Y. K. Tsai, "Job Shop Scheduling with a genetic algorithm and machine learning", International Journal of Production Research, vol. 35, no. 4, pp. 1171-1191, 1997.

[9] S. M. Sait and H. Youssef, VLSI Physical Design Automation: Theory and Pratice, McGraw-Hill, New York, 1995.

[10] M. H. Lin, "Modeling, Analysis, Prediction and Scheduling for Virtual Wafer Fabs", Ph.D. Thesis, Department of Computer Science \& Information Engineering, $\mathrm{Na-}$ tional Taiwan University, 2001.

[11] S. Y. Lin and H. P. Huang, "Modeling and Emulation of a Furnace in IC Fab Based on Colored-Timed Petri Net," IEEE Transactions on Semiconductor Manufacturing, vol. 11, no. 3, pp. 410-420, 1998. 